\title{
Análisis de competitividad del sistema de producción de lulo (Solanum quitoense Lam.) en tres municipios de Nariño
}

\section{Analysis of the competitiveness of the lulo production system (Solanum quitoense Lam.) in three municipalities of Nariño}

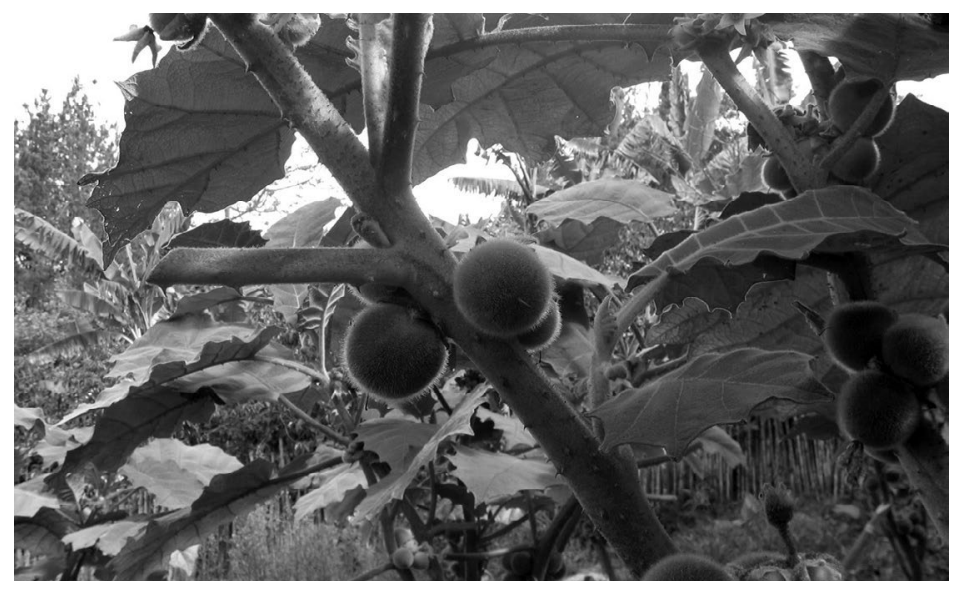

JOHANNA ALIXA MUÑOZ B. ${ }^{1}$

LUIS FELIPE RODRÍGUEZ C.2, 4

LILIA TERESA BERMUÚDEZ C. ${ }^{3}$

Cultivo de lulo en el departamento de Nariño.

Foto: H. Criollo

\section{RESUMEN}

El lulo es considerado un cultivo promisorio debido a su valor nutracéutico que lo hace apetecible en los mercados nacionales e internacionales. Colombia, por ser parte del centro de origen de esta especie frutal, cuenta con una oferta ambiental óptima para el cultivo y una amplia variabilidad; el aprovechamiento de estas ventajas, puede hacer que este cultivo sea competitivo. Actualmente el cultivo en Colombia presenta múltiples problemas que limitan su productividad y competitividad, ya que de un potencial calculado de $27,0 \mathrm{t} \mathrm{ha} \mathrm{a}^{-1}$ solamente se tiene un promedio nacional de $8,2 \mathrm{t} \mathrm{ha}^{-1}$. Esta condición ha llevado al abandono progresivo del cultivo y con el agravante en la pérdida de mano de obra y del nivel de vida de los productores. Este estudio pretende ofrecer elementos para un análisis de competitividad del sistema producción del lulo utilizando un diseño metodológico no experimental. Los municipios estudiados presentan cultivos en un rango altitudinal entre los $1.788 \mathrm{msnm}$ en la vereda La Florida, municipio de Colón y 2.480 en la vereda Botanilla del municipio de Cartago (Nariño, Colombia). En general las producciones son bajas, aunque el rendimiento promedio de la zona estudiada es en $8,5 \mathrm{~kg} /$ planta. Las plantas son producidas por los mismos agricultores y solo unos pocos las compran en viveros especializados.

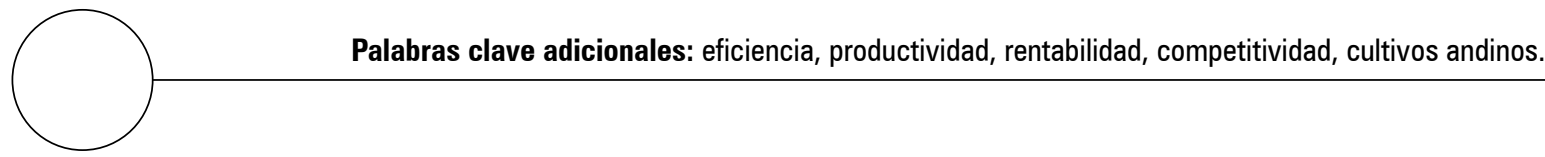

Facultad de Ciencias Agrarias, Universidad de Nariño, Pasto (Colombia).

Facultad de Ciencias Agrarias, Universidad Nacional de Colombia, Bogotá (Colombia).

Seccional Duitama, Universidad Pedagógica y Tecnológica de Colombia, Duitama (Colombia).

Autor para correspondencia.1frodriguezc@unal.edu.co 


\section{ABSTRACT}

Lulo is considered a promising crop because of its nutraceutical value, which makes it appealing to domestic and international markets. Colombia, forming part of this fruit's origin, has optimal environmental conditions for this crop and ample variability; taking advantage of these benefits could make this crop competitive. Currently, this crop has several problems in Colombia that limit its productivity and competitiveness with only $8.2 \mathrm{t} \mathrm{ha}^{-1}$ of the potential $27.0 \mathrm{t} \mathrm{ha}^{-1}$ being produced on average; leading to the ongoing abandonment of this crop and consequently a loss of labor and a lower quality of life for farmers. This study sought to provide the means for a competitive analysis of the lulo production system. A nonexperimental methodological design was used. The studied areas presented an altitudinal range for this crop of between 1,788 $\mathrm{m}$ a.s.1. in the La Florida district in the municipality of Colón and 2,480 $\mathrm{m}$ a.s.l. in the Botanilla district in the municipality of Cartago (Nariño, Colombia). In general, yields are low, although the average yield of the studied area was $8.5 \mathrm{~kg} /$ plant. The plants are produced by the farmers themselves and only a few are bought at a specialized nursery.

Additional key words: efficiency, productivity, profitability, competitiveness, Andean crops.

Una de las ramas productivas en las que se tiene un alto grado de confianza por las ventajas comparativas existentes es la fruticultura, dentro de la cual el lulo ocupa un lugar destacado, ya que es considerado un fruto exótico categorizado dentro del Acuerdo Competitividad de Productos Hortofrutícolas Promisorios Exportables en Colombia (Pinzón, 2003).

El cultivo de lulo es un renglón de gran importancia en el departamento de Nariño por su aporte en la generación de empleo, y por el mejoramiento del nivel de vida de los productores. Sin embargo, la problemática de este sistema productivo está ligada a múltiples factores relacionadas con aspectos de índole tecnológico, fitosanitario y de comercialización, los cuales amenazan su sostenibilidad (Angulo, 2008); ya son muchos los productores que han abandonado el cultivo por la reducción de su competitividad.
En general, el cultivo de lulo en Colombia se encuentra en zonas cafeteras, es de carácter minifundista, el promedio de plantas por agricultor no sobrepasa las 1.000 plantas y los niveles tecnológicos son limitados.

El término "competitividad" desde el punto de vista agrícola y en el marco del desarrollo sostenible, es un concepto conformado por aspectos sociales, políticos, ambientales y económicos, los cuales tienen como finalidad la equidad, gobernabilidad, sostenibilidad y rentabilidad, respectivamente (Rojas y Sepúlveda, 1999).

En el marco de desarrollo sostenible, el término competitividad de la agricultura se compone de cuatro dimensiones: social, políticamente-institucional, ambiental y económica, cuyos objetivos son la equidad, gobernabilidad, sostenibilidad y competitividad (Sepúlveda et al., 1998). 
A partir del proceso de globalización se ha hecho necesario, sobre todo en el nivel agrícola, crear estrategias que permitan permanecer en el mercado, mediante la ampliación de campos de acción que aseguren el cumplimiento de los objetivos de la rentabilidad (Chavarría y Sepúlveda, 2001).

Lascano (2002) realizó un estudio de competitividad del tomate de árbol en el Ecuador tomando como referencias las principales corrientes ideológicas sobre competitividad y cadenas agroalimentarias; en los lineamientos para determinar las ventajas competitivas, sus fuentes y factores determinantes, se tuvieron en cuenta los postulados de Michael Porter y las definiciones de competitividad recogidas por Rojas y Sepúlveda (1999), considerando las tendencias en las que proponen a la agricultura como un sector ampliado y la competitividad agroindustrial basada en la creación del valor al consumidor. Esta investigación concluye que la cadena del tomate de árbol en el Ecuador no es competitiva pero cuenta con potencialidades para llegar a serlo mediante la implementación de medidas y acciones planificadas por las entidades públicas y privadas.

Escudero (2002) realizó un estudio para evaluar la competitividad del sistema agroalimentario del tomate riñón en la década del noventa, en el Ecuador. Se enfatiza acerca de los problemas que se tienen para medir algunas variables como productividad y rentabilidad por la dificultad de acceso a una información válida y actualizada.

En el subsector agrícola colombiano, se puede destacar el estudio de competitividad de la cadena hortofrutícola del Valle del Cauca realizado en el marco del convenio IICA-SAC en el 2003 (Melo, 2005). Este estudio permitió obtener un diagnóstico de competitividad de la cadena, partiendo de la hipótesis de Sachs, que fundamenta el crecimiento económico en el cambio tecnológico. Analizando la relación entre la producti- vidad de los factores y su efecto sobre su competitividad, medida a partir de la relación entre los rendimientos esperados y los rendimientos promedios observados u obtenidos por los productores hortícolas del Valle del Cauca.

Otro trabajo importante es el realizado por Melo (2005), quien analiza la competitividad del sistema agroalimentario de la cebolla de bulbo, con enfoque de cadena productiva, en el distrito de riego del alto Chicamocha (Boyacá); este estudio estuvo orientado al análisis de competitividad bajo el enfoque de cadena productiva, haciendo énfasis en los eslabones de producción y comercialización, a nivel micro, donde los factores que se tuvieron en cuenta estaban constituidos por la capacidad de gestión, organización de los productores, desarrollo de la cadena productiva, cooperación tecnológica, recurso humano y el producto como determinantes de competitividad.

La medición de competitividad puede realizarse desde diversos enfoques y metodologías; incluso la unidad de análisis puede variar entre países y regiones, entre empresas, cadenas productivas y productos, dependiendo en cada caso de los intereses del estudio o programa en particular. Ante la variedad de perspectivas para medir la competitividad, se presenta el debate sobre cuáles indicadores son los más eficientes. Actualmente se discuten propuestas que adicionan nuevos rubros, restando importancia a la cuantificación y destacando el orden cualitativo sobre los factores externos (macro) (Sánchez, 2002).

Caro e Ibáñez (2006) evaluaron la competitividad en la agricultura de Sonora (México) siguiendo el enfoque de competitividad sistémica a nivel microeconómico. El cálculo de competitividad se realizó a partir de la empresa como unidad económica componente central de la cadena agroindustrial. El objetivo fue evaluar la competitividad en cada uno de los eslabones de la cadena, desde el productor hasta el distribuidor con el propósito de identificar los factores 
críticos de competitividad, que permitan proponer alternativas y diseñar políticas públicas y estrategias privadas, que favorezcan la capacidad competitiva de los diversos sectores productivos.

Según Sepúlveda et al. (1998), los indicadores más empleados para evaluar la competitividad a nivel micro en cadenas agroindustriales para los agentes participantes en cada eslabón, son: (1) relación precio/costo y (2) tasa de ganancia.

La competitividad de las agroempresas productoras de lulo en el contexto de la presente investigación, debe entenderse como la capacidad para mantener este producto en el mercado por largo tiempo, mediante el mejoramiento continuo de su tecnología para ofertarlo con calidad a un precio razonable para el consumidor.

El presente trabajo es un acercamiento a la medición de competitividad del sistema productivo de lulo de tres municipios del norte del departamento de Nariño, que permitirá facilitar la comprensión del porqué de la deserción de los cultivadores para dedicar sus tierras a otras actividades productivas aparentemente menos rentables como la ganadería o el café.

El objetivo general propuesto en este estudio fue analizar la competitividad del sistema de producción de lulo (Solanum quitoense Lam.) en los municipios de Cartago, Colón y San Lorenzo, departamento de Nariño, con el propósito de diseñar un plan estratégico para promover su mejoramiento y garantizar su sostenibilidad.

\section{METODOLOGÍA Y PROCEDIMIENTOS DE INVESTIGACIÓN}

El presente estudio responde a una investigación con diseño metodológico no experimental, de tipo descriptivo, cuya información primaria se acopio en el año 2012.
Se llevó a cabo en los municipios de Cartago, Colón y San Lorenzo ubicados al norte del departamento de Nariño donde se localiza el 28,8\% (156 ha) del área sembrada del cultivo de lulo del departamento (Secretaría de Agricultura y Medio Ambiente de Nariño, 2009).

La población objetivo (unidades de investigación) estuvo constituida por las agroempresas productoras de lulo de los municipios mencionados. Según los directores de las Umatas de estos municipios, las unidades productivas de lulo ascienden actualmente a un total de 215 , de las cuales 103 están ubicadas en Cartago, 42 en Colón y 70 en San Lorenzo. La unidad informante la constituyó el productor que lleva el manejo del sistema de producción del cultivo de lulo en su respectiva agroempresa.

El muestreo que se utilizó fue de tipo aleatorio estratificado con afijación proporcional por municipio y por tamaño de la unidad productiva, el cual fue determinado por el número de plantas en cada unidad de explotación.

El tamaño de muestra investigada se calculó a partir de las unidades productoras reportadas por las Umatas aplicando la fórmula propuesta por Kish (1982):

$n=\frac{z^{2} P Q N}{e^{2}(N-1)+z^{2}(1-P)}$

donde,

$\mathrm{N}=$ población (215 unidades productoras de lulo)

$Z=$ nivel de confianza (95\%)

$e=$ error muestral $(10 \%)$

$\mathrm{P}=$ proporción de unidades con una producción mayor que el promedio (30\%) 
$\mathrm{Q}=$ proporción de unidades con una producción menor que el promedio (70\%)

$\mathrm{n}=$ tamaño de muestra

El tamaño de muestra investigado fue de 59 unidades, las cuales se distribuyeron teniendo en cuenta la participación de cada municipio en número y tamaño de las explotaciones (tabla 1).

La fuente de la información primaria estuvo constituida principalmente por los productores de lulo ubicados en los municipios de Cartago, Colón y San Lorenzo, a quienes se les dirigió la aplicación de un formulario (encuesta) y por supuesto, la observación directa de las características técnico-económicas del sistema productivo de lulo realizada en las visitas.

Como fuentes de información secundaria, se utilizaron informes y estadísticas oficiales de la rama productiva de la fruticultura, provistas por el Ministerio de Agricultura y Desarrollo Rural, la Secretaría de Agricultura de Nariño y planes de desarrollo de cada municipio.

En la recolección de la información se emplearon: la consulta bibliográfica, el formulario, el diario de campo, las notas de entrevistas personales y la ubicación geográfica (GPS).

Con la información obtenida a través de la realización de la encuesta, se identificaron 59 variables cualitativas y 12 variables cuantitativas; debido a la necesidad de simplificar al máximo la dimensión de este elevado número de variables y con el ánimo de perder la menor cantidad de información posible, se utilizaron métodos de análisis multivariados soportados en el uso del software SPAD® 3.5 .

El cálculo de la rentabilidad se hizo según la fórmula propuesta por Sánchez (2002), teniendo en cuenta un periodo productivo del lulo de dos años y realizando previamente la actualización de los valores del año 2009, a partir de un índice de inflación del 3,17\% del año 2010.

Rentabilidad $=[(\operatorname{Rel} B / C)-1] * 100$

Igualmente se determinó el indicador competitividad precio/costo, el cual pretende resaltar la capacidad del agroempresario de lulo para obtener beneficios comparados con otros productores (otras agroempresas) por la vía de la formación de precios de mercado y los costos de producción. Este enfoque se relaciona con la teoría de competitividad de Porter (1991) que señala la existencia de dos formas de competitividad, una por costos y otra por diferenciación.

El indicador de competitividad (precio/costo) se calculó según la fórmula propuesta por Ibáñez y Caro (2000):

Competitividad precio/costo $=\frac{P x}{a P^{m}}$

Tabla 1. Distribución de las unidades de investigación que constituyen la muestra por municipio, según el número de unidades productivas y el tamaño de las explotaciones productivas (número de plantas).

\begin{tabular}{|c|c|c|c|c|}
\hline $\begin{array}{c}\text { Estrato } \\
\text { (plantas/unidad productiva) }\end{array}$ & Cartago & Colón & San Lorenzo & Total \\
\hline$<1.000$ & 12 & 4 & 12 & 28 \\
\hline $1.000-2.000$ & 12 & 5 & 6 & 8 \\
\hline$>2.000$ & 4 & 3 & 1 & 59 \\
\hline Total & 28 & 12 & 19 & 8 \\
\hline
\end{tabular}


donde,

$\mathrm{P}_{\mathrm{x}}=$ precio de la unidad del producto agrícola (tonelada de lulo)

$\mathrm{a}=$ insumos requeridos para producir una tonelada de lulo

$\mathrm{Pm}^{\mathrm{m}}=$ valor de los insumos requeridos para la producción de una tonelada de lulo

La "competitividad tasa de ganancia" determina el margen de beneficio basado en la eficiencia para obtener ganancia y se define como la diferencia entre las ventas totales y los costos totales (Ibáñez y Caro, 2000).

Igual que en el caso anterior, la competitividad tasa de ganancia para el sistema de producción de lulo, se calculó con base en la fórmula propuesta por Ibáñez y Caro (2000):

Competitividad tasa de ganancia $=\frac{P x-a P^{m}}{K p}$

donde,

$\mathrm{K}_{\mathrm{p}}=$ requerimiento de capital por tonelada producida de lulo

La información obtenida mediante el diario de campo y notas de entrevistas se tuvieron en cuenta para aclarar y complementar aspectos relacionados con la producción y el análisis de la información.

\section{ANÁLISIS Y DISCUSIÓN DE RESULTADOS}

Uno de los problemas prioritarios, común a todas las especies frutícolas, es la precaria zonificación asociada con la falta de conocimiento sobre los sistemas de producción, la oferta ambiental de los nichos actualmente utilizados y los requeri- mientos de los cultivos (Osorio, 2002). El lulo al igual que toda especie vegetal tiene sus propias exigencias y rangos de tolerancia respecto de los diferentes factores ambientales y socioeconómicos, lo que hace que se limite su área de distribución a aquellas zonas que presenten ventajas comparativas y competitivas.

Actualmente el área sembrada del cultivo de lulo en los municipios de Cartago, Colón y San Lorenzo asciende a 166 ha, cultivos localizados principalmente en 14 veredas.

Por la información obtenida mediante la encuesta se permitió establecer que el 55,9\% de los cultivos se ubican por encima de los $2.000 \mathrm{msnm}$, el $23,7 \%$ entre 2.000 y $1.900 \mathrm{msnm}$ y el $20,3 \%$ por debajo de los $1.900 \mathrm{msnm}$, condición muy relacionada con el tiempo del ciclo del sistema productivo de lulo requerido desde la siembra hasta la cosecha del producto. Predominan en la zona las explotaciones pequeñas con menos de 2.000 plantas $(86,4 \%)$, lo cual es causa de una mayor heterogeneidad en la producción y calidad, así como una desventaja en la capacidad de negociación durante el proceso de comercialización. En la mayoría de las unidades productivas el tipo de tenencia de la tierra son propietarios, el $81,4 \%$, el $5,1 \%$ son arriendo y el 13,5\% trabajan en compañía (parcería).

Los cultivos se programan haciendo coincidir la siembra con la época de lluvias en un 42,3\%, el $47,5 \%$ no hacen ningún tipo de programación y solamente el 10,2\% realizan siembras escalonadas que les permiten mantener su producción a través de todo el año. Las siembras de lulo se realizan principalmente en los meses de febrero, junio, octubre y noviembre, para aprovechar las épocas de lluvia y las principales cosechas se concentran en los meses de enero, febrero, octubre y noviembre, aunque en la zona siempre existe oferta del producto.

Por tratarse de un cultivo que no permite realizar siembras directas, la calidad de las plantas 
es de gran importancia para el desempeño de la plantación; el 89,8\% de los productores hacen sus propios semilleros y solamente el 10,2\% compran plantas producidas por viveros especializadas, en Popayán o La Unión.

El 47,5\% de los productores utilizan densidades de siembra menores a 2.000 plantas/ha, el $32,2 \%$ utiliza densidades entre 2.000 y 3.000 plantas/ ha, y el 20,3\% siembra más de 3.000 plantas/ha; esta situación repercute en diferentes grados la susceptibilidad a problemas sanitarios relacionados con enfermedades, ya que las altas densidades permiten mantener altos niveles de humedad, propicios para el desarrollo de enfermedades como la gota (Phytophthora infestans), pudrición algodonosa (Sclerotinia sclerotiorum) y antracnosis del fruto (Colectotrichum sp.), limitantes para la producción. La siembra de las plantas se fertiliza con solo abonos orgánicos en el 50,8\% de los casos, el $30,5 \%$ con solo abonos químicos y en mezcla el 18,6\%.

E1 $54,2 \%$ de los cultivadores aplican riego por aspersión, el 30,5\% lo aplican localizado utilizando mangueras convencionales y el $15,3 \%$ no aplican riego. Un $30,5 \%$ de los cultivadores aplican fertilizantes cada mes, un $64,4 \%$ lo hacen cada dos meses y un $5,1 \%$, cada 4 meses. Estas fertilizaciones las realizan aplicando solo abonos químicos $(74,6 \%)$ o mezclas de orgánicos con químicos (25,4\%). Los fertilizantes empleados son mayoritariamente los compuestos $(50,8 \%)$ y solo un $8,5 \%$ utilizan los simples o indiscriminadamente abonos compuestos y simples (40,7\%). Las cantidades aplicadas son variables y dependen de la edad del cultivo; en estado de planta adulta, un 81,4\% aplica $100 \mathrm{~g} /$ planta, el 8,5\% aplica $150 \mathrm{~g} /$ planta y el 10,1\% aplica $200 \mathrm{~g} /$ planta. Es importante anotar que dentro de la población encuestada ninguno realiza análisis de suelos, lo cual permite afirmar que las fertilizaciones se hacen por recomendaciones muy generales, sin tener en cuenta el estado nutricional del suelo ni los requerimientos de la planta.
En relación al control de malezas, en la zona se realiza con métodos mecánicos (30,5\%), manuales $(18,6 \%)$ e integrados de control $(50,8 \%)$; predominan el uso de guadañadoras y su combinación en algunos casos con químicos, prácticas que son favorables para evitar el deterioro erosivo del suelo. Los problemas fitosanitarios son los más serios que afrontan las agroempresas de lulo en la zona norte de Nariño; las plagas igualmente son determinantes de la productividad del lulo y de su manejo depende en gran medida la calidad del producto.

El control de los problemas mencionados anteriormente se hace a partir de productos químicos exclusivamente $(76,3 \%)$ y mediante la combinación de químicos con algunas prácticas culturales como las podas o en el caso de las plagas con la instalación de trampas (23,7\%).

Los productores de la zona realizan las cosechas cada 15 d (50,8\%), cada $21 \mathrm{~d}(40,7 \%)$ y cada 30 d $(8,5 \%)$; las cosechas frecuentes son un factor importante en la definición de la uniformidad en los puntos de maduración, lo cual es clave en la clasificación. La clasificación del lulo en la zona se hace principalmente por tamaño (78\%), lo cual es importante a tener en cuenta cuando se programan nuevos cultivos. Genotipos con altas producciones de frutos pequeños, como el lulo 'La Selva', tendrían problemas de comercialización, principalmente porque las categorías de precio se basan en el tamaño de la fruta.

La presentación de la fruta es de gran importancia en la comercialización; sin embargo solo el $11,9 \%$ de los productores dicen limpiar la fruta, mientras que el resto afirma que la fruta se depila durante el proceso de empaque y transporte. Igualmente, el método de cosecha es determinante de la calidad; mientras que el 37,3\% dice utilizar baldes plásticos y el 11,9\% canastillas, todavía existe un alto porcentaje de productores que cosechan en costal $(50,8 \%)$, incrementando así los daños físicos por aplastamiento. Otro aspecto determinante de la ca- 
lidad poscosecha es el tipo de transporte desde el sitio de cosecha al lugar de venta; el 54,2\% transporta el lulo en costales y al hombro, el $37,3 \%$ lo hace en caballo y solamente el $8,5 \%$ utiliza medios mecanizados.

Los mayores problemas que afrontan los productores de lulo del norte de Nariño están relacionados con dificultades económicas y el difícil acceso al crédito (69,5\%), y la consecución de mano de obra, especialmente en épocas de cosecha de café $(10,2 \%)$. El número de jornales es una variable poco identificada en la zona, ya que se utiliza mucha mano de obra familiar (productor, esposa, hijos) y es un aspecto que el productor normalmente no contabiliza como gasto. E1 análisis de la encuesta permitió establecer que el $47,5 \%$ utilizan menos de 250 jornales/ha al año, el $30,5 \%$ emplea entre 250 y 300 jornales/ha al año y el 22,0\% requiere más de 300 jornales/ha al año. Estos jornales son en su mayoría hombres (91,5\%) y solamente el $8,5 \%$ es mano de obra femenina. Predomina el sistema de jornal libre (con alimentación) sobre el jornal grabado (sin alimentación) con un $66,1 \%$ y $33,9 \%$, respectivamente.

La comercialización del lulo en la zona se hace principalmente a través de intermediarios que recogen la fruta en la finca para transportarla a las centrales de abasto mayorista de Pasto y Cali, principalmente.

En la zona existen cinco comercializadores de los cuales tres son esporádicos, que solo compran el producto cuando el margen de ganancia es significativo; los otros dos son permanentes y compran el producto que salga de la zona, así las condiciones de comercialización del producto no sean las mejores.

Según Sáenz (2008) se deben identificar cinco ejes principales de competitividad dentro de una empresa, la cual para las unidades productoras de lulo se hace explicita así:
Talento humano. La agroempresa de lulo mantiene el tradicional uso de mano de obra de mano familiar, con un nivel educativo bajo con prevalencia de trabajadores con educación primaria $(89,8 \%)$ y $\sin$ estudios $(10,2 \%)$, pero con vocación agrícola y dedicación al cultivo por más de cinco años (84,7\%).

El mercadeo. El fruto de lulo de la zona se diferencia de otras regiones por su color y acidez característico que lo hacen muy apetecido por el cliente, aunque en el momento de la comercialización del producto la clasificación la hace el comprador de acuerdo con su integridad física y tamaño.

El ingreso de altos volúmenes de fruta desde el Ecuador, afectan ostensiblemente los precios en el mercado de Cali, sitio donde se vende la producción del norte de Nariño. Este lulo es de menor calidad que el colombiano.

Oferta de valor de la organización. Las ventajas de la comercialización son para quienes tienen una mayor oferta de producto, por cuanto el intermediario recoge el producto directamente en la agroempresa y asume los costos de transporte. El agroempresario con oferta reducida debe llevar directamente la producción al sitio de acopio asumiendo el gasto de transporte. Es de destacar que en este aspecto un alto porcentaje de agroempresarios cultiva menos de 1.000 plantas $(47,5 \%)$.

Gestión. Hace referencia a todo lo que el agroempresario debe manejar para que la empresa cumpla con los objetivos propuestos; debe tener en cuenta actividades como la fijación de objetivos, formulación de estrategias para lograrlos, organización de tareas, actividades y personas; motivar y comunicar, controlar, evaluar. En este punto se tienen grandes falencias. El 100\% de los agroempresarios de lulo no llevan ningún registro ni control de las actividades desarrolladas en el cultivo. 
Capital de relaciones sociales. Las agroempresas deben tener la capacidad de realizar alianzas estratégicas y trabajar con organizaciones que les permitan beneficiarse mutuamente.

El análisis de la productividad de los sistemas productivos de lulo por municipio, expresada en $\mathrm{kg} /$ planta permite afirmar que en el municipio de Colón se encuentra un mayor número de explotaciones con rendimientos superiores a 7,0 $\mathrm{kg} /$ planta, representando un 58,3\% del total; el $41,7 \%$ restante se encuentra en la categoría que produce entre 4,0 y $7,0 \mathrm{~kg} /$ planta. En el municipio de Cartago, el 7,1\% de las unidades productoras presentan rendimientos inferiores a $4,0 \mathrm{~kg} /$ planta, el $50,0 \%$ producen entre 4,0 y $7,0 \mathrm{~kg} /$ planta; el $42,9 \%$ obtienen rendimientos superiores a $7,0 \mathrm{~kg} /$ planta (tabla 2). En el municipio de San Lorenzo, el 10,5\% de los productores obtienen rendimientos inferiores a $4,0 \mathrm{~kg} /$ planta, el $68,4 \%$ alcanzan una producción entre 4,0 y 7,0 $\mathrm{kg} /$ planta, mientras que el $21,1 \%$ logran rendimientos superiores a $7,0 \mathrm{~kg} /$ planta (tabla 2).

Al relacionar la productividad con el número de plantas que posee cada agricultor, se estableció que el $67,8 \%$ de los productores que poseen menos de 1.000 plantas obtienen rendimientos inferiores a 7,0 kg/planta; son agricultores con bajos niveles de inversión, con sistemas tradicionales de cultivo, bajos niveles tecnológicos y deficiente manejo de plagas y enfermedades. Las agroempresas más eficientes (más de $7,0 \mathrm{~kg} /$ planta) se encuentran entre las que poseen más de 2.000 plantas $(75,0 \%)$. Normalmente, las unidades productivas con más plantas, poseen una mayor capacidad de inversión y alto nivel tecnológico que las hace más productivas (tabla 2).

El análisis de la TIR (tabla 3) mostró igualmente la bondad económica de las agroempresas que trabajan con mayor número de plantas $(4.000$ plantas), poseen las mayores áreas de cultivo (3,0 ha) y están ubicadas en un rango altitudinal inferior (1.936 msnm).
El sistema de producción de lulo de la zona norte de Nariño es muy atractivo desde el punto de vista financiero; sin embargo, se hace necesario resaltar los altos riesgos de la inversión, considerando la susceptibilidad de los genotipos regionales a enfermedades muy limitantes como la gota ( $P$. infestans), marchitamiento vascular (Fusarium oxysporum), nematodos e insectos, que unidos a cambios climáticos pueden causar la pérdida total del cultivo.

Entre los indicadores que permiten una medición aproximada de competitividad de las agroempresas objeto de la investigación se destacan la productividad y el porcentaje de participación en el mercado; el primero se puede comparar con las productividades nacional y departamental. En este sentido se observa que de una producción nacional de 52.147 t, 2.913 t son producidas en el departamento de Nariño y 833 t en los municipios estudiados, lo cual representa un 5,48\% y $1,57 \%$, respectivamente; al comparar la producción departamental con la de los municipios analizados, estos representan un $28,6 \%$, destacándose su condición de municipios productores en el ámbito departamental (tabla 4). La productividad parcial de la tierra (rendimiento por hectárea) a nivel de la zona de estudio $\left(8,5 \mathrm{t} \mathrm{ha}^{-1}\right)$ es superior al promedio nacional $\left(8,2 \mathrm{t} \mathrm{ha}^{-1}\right) \mathrm{y}$ está muy por encima del promedio departamental que se estima en 5,3 t ha-1 (Agronet, 2009); esta característica permite establecer la vocación lulera y la especialización que han adquirido en el cultivo los productores de estos municipios del norte de Nariño.

El área sembrada y su participación en producción con respecto a los valores nacionales y departamentales son una forma indirecta de medir la participación en el mercado como un indicador de competitividad. En cuanto al área sembrada, el departamento de Nariño presenta un total de 554 ha de lulo de las 6.356 ha reportadas a nivel nacional representando el 8,71\% (Agronet, 2009). La zona de estudio representa el $29,9 \%$ del 
Tabla 2. Interacciones entre algunas variables estratégicas con la productividad de una planta de lulo (resumen de la tabla de Burt).

\begin{tabular}{|c|c|c|c|}
\hline Municipio & Menos de 4 kg/planta & Entre 4 y 7 kg/planta & Más de 7 kg/planta \\
\hline Cartago & 7,1 & 50,0 & 42,9 \\
\hline Colón & 0,0 & 41,7 & 58,3 \\
\hline S. Lorenzo & 10,5 & 68,4 & 21,1 \\
\hline \multicolumn{4}{|c|}{ No. plantas/unidad productiva } \\
\hline$<1.000$ plantas & 7,1 & 60,7 & 32,1 \\
\hline $1.000-2.000$ plantas & 8,7 & 56,5 & 34,8 \\
\hline$>2.000$ plantas & 0,0 & 25,0 & 75,0 \\
\hline \multicolumn{4}{|l|}{ Densidad } \\
\hline$<2.000$ plantas/ha & 7,1 & 50,0 & 42,9 \\
\hline 2.000-3.000 plantas/ha & 10,5 & 63,2 & 26,3 \\
\hline$>3.000$ plantas/ha & 0,0 & 50,0 & 50,0 \\
\hline \multicolumn{4}{|l|}{ Fertilización } \\
\hline $100 \mathrm{~g} /$ planta & 6,2 & 56,2 & 37,5 \\
\hline $150 \mathrm{~g} /$ planta & 20,0 & 40,0 & 40,0 \\
\hline $200 \mathrm{~g} /$ planta & 0,0 & 50,0 & 50,0 \\
\hline \multicolumn{4}{|l|}{ Experiencia } \\
\hline De 2 a 5 años & 11,1 & 66,7 & 22,2 \\
\hline$>5$ años & 6,0 & 52,0 & 42,0 \\
\hline
\end{tabular}

Tabla 3. Flujo neto de caja (en miles de pesos) TIR y RB/C de los grupos de agroempresas productoras de lulo resultantes del análisis de clasificación (calculados con base en 1 ha).

\begin{tabular}{|l|r|r|r|r|r|r|r|r|}
\hline & \multicolumn{2}{|c}{ Grupo 1 } & \multicolumn{2}{c|}{ Grupo 2 } & \multicolumn{2}{c|}{ Grupo 3 } & \multicolumn{2}{c|}{ Grupo 4} \\
\cline { 2 - 10 } & \multicolumn{1}{|c|}{ Año 1 } & \multicolumn{1}{c|}{ Año 2 } & \multicolumn{1}{c|}{ Año 1 } & Año 2 & Año 1 & \multicolumn{1}{c|}{ Año 2 } & Año 1 & Año 2 \\
\hline Costo total & $2.978,5$ & $4.061,5$ & $3.834,1$ & $3.137,0$ & $4.177,6$ & $3.418,6$ & $4.047,5$ & $3.311,6$ \\
\hline Ingresos & 0,0 & $19.000,0$ & $1.312,5$ & $16.187,5$ & 2.133 .3 & $26.644,4$ & $5.333,3$ & $34.666,7$ \\
\hline Flujo neto & $-4.964,2$ & $14.938,5$ & $-2.521,6$ & $13.050,5$ & $-2.044,2$ & $23.226,4$ & $1.285,8$ & $31.355,0$ \\
\hline TIR (\%) & & 31,0 & & 55,0 & & 113,0 & & 195,0 \\
\hline RB/C & & 1,2 & & 1,6 & & 2,7 & & 4,9 \\
\hline
\end{tabular}

área departamental de 166 ha, lo cual reafirma la importancia de esta región del norte de Nariño como cultivadora de lulo.

Otros indicadores de competitividad son los de precios y costos, los cuales permiten comparar los precios promedios anuales en diferentes regiones del país con el precio doméstico, que en este caso se considera el de la ciudad de Cali, por ser este mercado el principal regulador de los pre- cios de compra en la zona productora del norte del departamento de Nariño. Se estableció que los precios de Cali son muy competitivos y es un mercado relativamente cercano a la zona estudiada; mercados más distantes y con mayores dificultades para el transporte, como Neiva y Armenia, presentan precios inferiores al mercado de Cali; los mayores precios alcanzados por el lulo se dan en mercados más alejados como Barranquilla, Medellín y Bogotá (tabla 5), los cua- 


\begin{tabular}{|l|c|c|}
\hline Tabla 4. Indicadores de competitividad de las agroempresas productoras de lulo en los municipios de Cartago, \\
Colón y San Lorenzo (Nariño, Colombia).
\end{tabular}

Fuente: Agronet (2011), Secretaría de Agricultura y Medio Ambiente de Nariño (2009).

les implican para el productor un mayor riesgo de deterioro de la fruta y costos de transporte significativamente más altos.

En cuanto a los costos de producción se estableció que estos son significativamente más bajos en la zona de estudio comparados con los costos de otras regiones productoras en Colombia (tabla 5), lo cual puede deberse principalmente a dos condiciones: la menor densidad de siembra utilizada en la región (1.333 plantas/ha), que implica un menor costo en plántulas, fertilizantes, pesticidas, plateos, riego, podas y deschuponadas y en general en todas las labores relacionadas con el cultivo; otra razón que favorece los menores costos de producción de lulo en la región estudiada es el valor del jornal, que no supera para el año 2010, los $\$ 10.000 /$ jornal.

La estimación de competitividad basada en los cálculos de rentabilidad, competitividad precio/costo y competitividad tasa de ganancia para cada uno de los grupos de agroempresas conformados según el análisis clasificatorio, se incluyen en la tabla 6.

Es importante anotar que a pesar de que el sistema de cultivo de lulo en el norte de Nariño muestra valores de competitividad entre aceptables a altos, la problemática del abandono del cultivo existe, por los grandes esfuerzos que deben hacer los agroempresarios para sacar los cultivos

Tabla 5. Indicadores de competitividad relacionados con el precio promedio anual de venta y los costos de producción del cultivo de lulo.

\begin{tabular}{|c|c|c|c|}
\hline \multicolumn{2}{|c|}{$\begin{array}{l}\text { Precio promedio anual en principales centros de abastos de } \\
\text { Colombia (2011) }\end{array}$} & \multicolumn{2}{|c|}{$\begin{array}{l}\text { Costos de producción/ha por ciclo de producción de las } \\
\text { principales zonas productoras de lulo en Colombia (2011) }\end{array}$} \\
\hline Mercado & Precio/kg (\$) & Región & Costos (\$) \\
\hline Cali & 2.040 & $\begin{array}{l}\text { Zona norte de Nariño } \\
\text { (1.333 plantas/ha) }\end{array}$ & 7.573 .517 \\
\hline Pasto & 1.742 & $\begin{array}{l}\text { Valle del Cauca } \\
\text { (3.330 plantas/ha) }\end{array}$ & 14.557 .235 \\
\hline Neiva & 1.793 & Tolima (3.330 plantas/ha) & 20.161 .269 \\
\hline Armenia & 2.004 & Huila (1.666 plantas/ha) & 12.989 .046 \\
\hline Manizales & 2.189 & & \\
\hline Medellín & 2.277 & & \\
\hline Bogotá & 2.237 & & \\
\hline Barranquilla & 3.405 & & \\
\hline
\end{tabular}

Fuente: Agronet (2011), Secretaría de Agricultura y Minería del Huila (2011). 


\begin{tabular}{|c|c|c|c|c|}
\hline Indicador & Grupo 1 & Grupo 2 & Grupo 3 & Grupo 4 \\
\hline Rentabilidad (\%) & 122,60 & 156,80 & 269,70 & 488,40 \\
\hline Competitividad precio/costo & 5,55 & 7,30 & 10,99 & 15,89 \\
\hline Competitividad tasa/ganancia & 1.73 & 2.17 & 3,32 & 5,40 \\
\hline
\end{tabular}

adelante, debido principalmente a problemas de índole sanitaria.

\section{CONCLUSIONES}

En los cultivos de lulo subsisten problemas relacionados con la baja tecnología característica de las economías campesinas. Entre los principales problemas se destacan el tamaño de las explotaciones, el $86,5 \%$ poseen menos de 2.000 plantas, ningún productor realiza análisis de suelo, el 78\% no aplica microelementos y predominan los sistemas de riego por aspersión de carácter rudimentario.

La baja productividad parcial de la tierra está estrechamente asociada con el bajo nivel tecnológico. La alta inversión por planta, unida al alto costo por tonelada de fruta producida es predominante en la región. Aquellos productores con bajos costos de tonelada producida están asocia- dos a un mayor número de plantas, obtienen los mayores ingresos y una alta rentabilidad, lo cual los hace más competitivos.

En general, la productividad de los cultivos es relativamente baja ya que en el $57,6 \%$ de las unidades productivas se obtienen rendimientos inferiores a $7,0 \mathrm{~kg} /$ planta, muy por debajo de su potencial.

La competitividad de las agroempresas se ve afectada por factores internos y externos asociados a la formación del precio del fruto, el cual es determinado por el intermediario teniendo en cuenta el tamaño del fruto.

La asociación de las agroempresas permitiría coordinar las diferentes funciones de la cadena productiva y generar ventajas competitivas en los asociados, frente a quienes lo hacen individualmente.

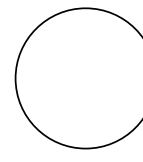

Agronet. 2009. Área cosechada, producción y rendimiento de lulo, 2009. En: http://www.agronet.gov. co.; consulta: 24 de julio de 2013.

Agronet, 2011. Área cosechada, producción y rendimiento de lulo, 2011. En: http://www.agronet.gov. co; consulta: 18 de agosto de 2013.

Angulo, R. 2008. El cultivo de lulo. Colciencias, Universidad Jorge Tadeo Lozano, Bogotá.

Caro, J. y C. Ibañes. 2006. Competitividad en la agricultura de Sonora. Análisis de la competitividad

\section{REFERENCIAS BIBLIOGRÁFICAS}

sistémica para el nivel micro de cinco cultivos. En http://portalescolson.com/boletines/276/Agricultura $\% 20$ en $\% 20$ Sonora.pdf; consulta: 23 de febrero del 2011.

Chavarría, H. y S. Sepúlveda. 2001. Factores no económicos de la competitividad. Cuaderno Técnico No. 18. Competividad de la Agricultura: Cadenas Agroalimentarias y el Impacto del Factor Localización Espacial. IICA, San José.

Escudero, A. 2002. Evaluación de la competitividad del sistema agroalimentario del tomate de riñón: pro- 
ducción de la década de los 90’s. Trabajo de grado, Facultad de Economía. Pontificia Universidad Católica del Ecuador (PUCE), Quito.

Ibáñez, C. y J. Caro. 2000. Algunas teorías e instrumentos para el análisis de la competitividad. Cuaderno Técnico No. 15. Competividad de la Agricultura: Cadenas Agroalimentarias y el Impacto del Factor Localización Espacial. IICA, San José.

Kish, L. 1982. Muestreo de encuestas. $3^{a}$ reimpresión. Trillas, México DF

Lascano, V. 2002. Análisis de competitividad de la cadena agroalimentaria del tomate de árbol en Ecuador. Trabajo de grado. Facultad de Economía, Pontificia Universidad Católica del Ecuador (PUCE), Quito.

Melo, L. 2005. Competitividad del sistema agroalimentario de la cebolla de bulbo, con enfoque de cadena productiva, en el distrito de riego del Alto Chicamocha-Boyacá. Tesis de maestría. Facultad de Agronomía, Universidad Nacional de Colombia, Bogotá.

Osorio, J. 2002. Plan de investigación y transferencia de tecnología para la fruticultura colombiana. Programa Nacional de Manejo Integrado de Plagas. Corpoica, Mosquera, Colombia.

Pinzón, 2003. Innovación, desarrollo tecnológico y competitividad en sistemas y cadenas de producción y clusters. Universidad la Gran Colombia, Armenia, Colombia.

Porter, M. 1991. Towards a dynamic theory of strategy. Strat. Manag. J. 12, 95-117.
Rojas, P. y S. Sepúlveda. 1999. خ̇Qué es la competitividad? Cuadernos Técnicos No. 9. Competitividad de la agricultura: cadenas agroalimentarias y el impacto del factor localización espacial. IICA, San José

Sáenz, U. 2008. Inteligencia económica y tecnológica: factor de innovación, competitividad y desarrollo. Tesis de maestría. Facultad de Ciencias Sociales y Humanidades, Universidad Autónoma Metropolitana de México, México D.F.

Sánchez, J. 2002. Análisis de rentabilidad de la empresa. En: 5campus.com, Análísis Contable, http:// www.5campus.com/leccion/anarenta; consulta: octubre de 2013

Secretaría de Agricultura y Medio Ambiente de Nariño. 2009. Consolidado Agropecuario de Nariño. San Juan de Pasto, Nariño. Gobernación de Nariño. En: http:// www.narino.gov.co/index.php?.option $=$ com remo sitory\&Itemid $=232 \&$ func $=$ startdown\&id $=2815$; consulta: 20 de agosto de 2011

Secretaría de Agricultura y Minería del Huila. 2009. Anuario Estadístico Agropecuario. Neiva Huila. pp. 305. En: http://www.huila.gov.co/documentos/A/ ANUARIO\%20ESTAD\%C3\%8DSTICO\%20 AGROPECUARIO\%20DEL\%20HUILA\%202009. pdf; consulta: septiembre de 2013.

Sepúlveda, S., A. Castro y P. Rojas. 1998. Metodología para estimar el nivel de desarrollo sostenible en espacios territoriales. Cuadernos Técnicos No. 4. IICA, San José. 